\title{
Foreword: the many fascinating functions of SMC protein complexes
}

\author{
Christian H. Haering
}

Published online: 20 March 2009

(C) Springer Science + Business Media B.V. 2009

Ever since the first description of mitotic chromosomes by Walther Flemming more than 125 years ago, researchers have been fascinated by the dynamic changes these gigantic assemblies of nucleic acids and proteins undergo during the cell cycle. How does the diffuse mass of interphase chromatin get organized into pairs of compact filamentous chromatids in preparation for cell division? What causes the sudden and synchronous separation of all chromatid pairs just before a cell divides, and how does it come about that the chromatids of every pair always move to opposite cell poles? What prevents the chromatids from getting entangled during this process and torn into pieces? And if chromosomes break, what is required to put them back together?

It was not until recently that a family of multisubunit protein complexes was found to be one of the keystones to providing answers to all the questions. The hallmark of these protein complexes is a pair of ATPase subunits that fold into an extended rodshaped structure and are now referred to as Structural Maintenance of Chromosomes (SMC) proteins. Their name reflects their fundamental roles in multiple aspects of chromosome metabolism. In eukaryotes, six different SMC proteins form the cores of three

Responsible Editor: Herbert Macgregor, Christian Haering

C. H. Haering $(\bowtie)$

European Molecular Biology Laboratory (EMBL),

Heidelberg, Germany

e-mail: christian.haering@embl.de discrete complexes. SMC2 and SMC4 together with three non-SMC subunits assemble into the condensin complex, which is indispensable for mitotic chromosome structure. SMC1 and SMC3 associate with two additional proteins to make the cohesin complex, which physically holds sister chromatids together and thereby ensures their segregation to opposite spindle poles. In addition, there is now a substantial body of evidence that cohesin and condensin-related complexes function in regulating gene transcription. Finally, SMC5 and SMC6 are the central subunits of a yet unnamed complex that has crucial roles in DNA repair and recombination. In prokaryotes, a single SMC complex seems to achieve different functions of its eukaryotic counterparts during nucleoid segregation. All species also posses a distinct complex essential for DNA repair that contains the SMC-like protein RAD50.

In this special issue of Chromosome Research, we have, for the first time, assembled a series of review and original articles that highlight the impressive manifold of vital functions that SMC protein complexes perform. We start with a review article on condensin, architect of mitotic chromosomes, by Damien Hudson, Kathryn Marhall, and William Earnshaw. Condensin's SMC2 subunit was first found as one of the most abundant proteins that remained associated with mitotic chromosomes after high salt extraction. Depletion of condensin SMCs from frog egg mitotic extracts prevented the formation of condensed chromosomes when chromatin was added 
to such extracts, hence the complex formed by the two SMCs and the three associated proteins was termed condensin. The recent findings that cells depleted of condensin subunits show a practically normal degree of prophase chromosome compaction, yet fail to segregate their chromosomes normally, have altered our view of condensin. Condensin may not be the actual motor driving chromosome condensation as initially believed, but is more likely the stabilizer that keeps mitotic chromatids in shape while they are being segregated to the cell poles.

What, if not condensin, might be the mysterious activity that propels chromosome condensation? The discovery of this activity will require new technologies for following chromosome condensation in living cells. Gabriel Neurohr and Daniel Gerlich evaluate the amazing methods that have now become available to quantitatively measure biophysical properties of chromosomes in vivo while cells undergo mitosis with a focus on state-of-the-art real-time fluorescence microscopy.

The two articles that follow take advantage of two of such cutting edge imaging techniques to study the chromosomal association of the second SMC complex, cohesin. Adrian McNairn and Jennifer Gerton use Fluorescence Loss In Photobleaching (FLIP) to determine the turnover dynamics of cohesin subunits on yeast chromosomes at different stages of the cell cycle. They find that cohesin proteins are surprisingly dynamic, coming on and going off interphase chromosomes every one or two minutes. Chromosomal cohesin turnover slows down as cells enter mitosis, especially at locations that most likely correspond to centromeric regions, but the half-life of cohesin on mitotic chromosomes is still only a fraction of the time the complex has to function in chromosome cohesion. How a complex that has to stably hold sister chromatids together can be so dynamic will be one of the exciting questions for the future.

Not only the development of new imaging technologies but also the use of novel model systems will be the key to revealing the functions of SMC complexes. Michel Bellini and colleagues introduce lampbrush chromosomes, the gigantic chromosomes present in oocyte nuclei during meiotic prophase, to study the localization and dynamics of cohesin. Fluorescence Recovery After Photobleaching (FRAP) measurements show that chromosomal cohesin in Xenopus meiotic oocytes turns over with similar kinetics to yeast cohesin. Strikingly, cohesin localization is detectable only at regions where the sisters chromatids are paired, while it is apparently absent from the large chromosomal loops that are actively being transcribed. This finding underlines the connection between cohesin and gene regulation, an emerging topic covered by the next two articles.

The review article by Dale Dorsett starts with the discovery that a protein called Nipped-B, which is required to load cohesin onto chromosomes, also has a role in transcriptional regulation in Drosophila. Importantly, not only mutations in the Nipped-B gene but also in genes encoding subunits of the cohesin complex lead to malformations in the fly, most likely by altering the expression of key developmental genes. The function of cohesin in transcriptional regulation second to its function in cohesion is conserved through evolution, since mutations in the human orthologues of Nipped-B and cohesin have been linked to developmental disorders. How cohesin influences the transcription of genes is not yet known, but one possibility is that cohesin might regulate longrange enhancer-promoter interactions.

Such a scenario is supported by the recent finding that cohesin co-localizes on human chromosomes with the transcriptional insulator protein CTCF. In their article, Kerstin Wendt and Jan-Michael Peters summarize the current evidence that cohesin is required for the enhancer-blocking activity mediated by CTCF and present attractive models that could explain this function in mechanistic terms.

Cohesin is not the only SMC complex that functions in gene regulation. In Caenorhabditis elegans, a multi-subunit protein complex that shares remarkable homology with the canonical condensin complex is essential to downregulate transcription from the two $\mathrm{X}$ chromosomes in hermaphrodites to match the expression levels from the single $\mathrm{X}$ chromosome in males. Sevinc Ercan and Jason Lieb discuss what is known about how this Dosage Compensation Complex (DCC) can fine-tune transcription by a factor of 2 over a very large domain, e. g., a whole chromosome. Key to understanding this fascinating mechanism may be finding out how the DCC is specifically targeted to the X chromosomes.

Another example of the recruitment of an SMC complex to specific chromosomal loci is the localization of cohesin to heterochromatic regions, which is the focus of the next review article by Marc 
Gartenberg. Among such regions are the silenced mating-type cassettes in budding yeast, where cohesin holds sister chromatids together in a manner that depends on transcriptional silencing of these loci. In fission yeast and vertebrates, cohesin is also strongly enriched at pericentromeric heterochromatin regions. Even though the mechanisms that ensure high levels of cohesin close to the centromeres may differ between organisms, they seem to be essential to ensure the formation of centromeric cohesion that is strong enough to resist the pulling forces of the mitotic spindle.

Centromeric cohesion also plays a central role to enable two rounds of chromosome segregation during meiosis. In their article, Takeshi Sakuno and Yoshinori Watanabe propose that two pools of cohesin, one at the core centromeres and one at pericentromeric regions, play distinct roles in the co-orientation of sister kinetochores to the same spindle pole during the first meiotic division and maintenance of centromeric cohesion until the second meiotic division. These different functions of cohesin around the centromere are achieved by its interplay with a regulatory protein named shugoshin that recruits protein phosphatase $2 \mathrm{~A}$, or with the so-called monopolin proteins.

Although the cellular roles of cohesin and condensin have been precisely defined, the exact molecular functions of the third eukaryotic SMC protein complex are yet to be revealed. Giacomo De Piccoli and Luis Aragon provide a comprehensive overview of the many involvements of the complex containing SMC5 and SMC6 proteins in various aspects of DNA damage repair and recombination. The fact that this complex contains the largest variety of different subunits of all SMC complexes, including one that possesses sumoylation activity, make it a fascinating yet challenging object for future studies.

An increasing amount of data suggests that the single SMC complex that exists in nearly all prokaryotes integrates most of the functions of its three eukaryotic counterparts. It is essential for correct chromosome partitioning and compaction during cell divisions, and it is connected to DNA repair path- ways. Peter Graumann and Tobias Knust summarize what is known about SMC complexes in Bacillus subtilis and other bacteria and discuss how the changes in DNA conformation that these proteins promote in vitro could explain their functions in vivo. Prokaryotes also posses other proteins that share architectural features with canonical SMCs and are involved in DNA damage repair.

Another universal protein complex that contains an SMC-like subunit called RAD50 is also a central player in the repair of DNA double strand breaks. While RAD50 homodimers contain ATPase domains and long coiled-coil domains that are more or less identical to classical SMCs, they differ in the way their coiled coils dimerize. Upon binding to chromosomes, the orientation of the RAD50 coiled coils changes substantially to an arrangement that is thought to act as 'molecular Velcro' for tethering broken DNA ends together. The review by Eri Kinoshita, Eddy van der Linden, and Claire Wyman centers on the mechanistic effects that ATP binding and hydrolysis by RAD50 and other SMC proteins can have on the molecular architecture of the complexes and their functions on chromosomes.

While the study of SMC protein complexes is still largely in its infancy, life without them now hardly seems possible. From bacteria to humans, all organisms depend on SMCs for passing on their genomes from one generation to the next. Finding out how the different complexes achieve such a variety of essential functions will be an exciting challenge for the future. I hope that after reading the articles in this issue you will share some of the fascination for this class of proteins that has now entered the center stage of chromosome biology.

I would like to thank all authors for their excellent contributions to this special issue, all referees for their valuable suggestions on the manuscripts, and Petra Riedinger for help with the cover art. My special thanks go to Herbert Macgregor, Editor-in-Chief of Chromosome Research, and his editorial board for their enthusiasm and support for this project. 